\title{
On the controllability of nonlinear fractional system with control delay
}

\author{
Musarrat Nawaz*(D), Jiang Wei ${ }^{\dagger}(\mathbb{D}$, Jiale Sheng (D), Azmat Ullah Khan Niazi (D), \\ LiChang Yang (i) \\ School of Mathematical Sciences, Anhui University, Hefei, Anhui, China, Hefei, 230039, China
}

\begin{abstract}
We discuss the controllability of nonlinear fractional control system with control delay. Firstly we obtain result about controllability of a linear fractional control system. After that, we give sufficient condition for the controllability of nonlinear fractional system with control delay. Our approach is based on Schauder fixed point theorem. At the end numerical example is constructed to support the result.
\end{abstract}

Mathematics Subject Classification (2010). 34K37, 34B15

Keywords. controllability, nonlinear system, control delay

\section{Introduction}

The fractional differential equations have attracted increasing attention in the past three decades, since it is a very useful and valuable tool in modeling the dynamics of processes through complex media in so many different applied topics, for details see $([6,8,9,18,19,21,26,27])$. Many mathematicians, engineers and physicists have their contributions in fractional differential equations theory and in its applications. Fractional derivatives have several kinds, such as Caputo, Riemann-Liouville, Grunwald-Letnikov and Hadamard etc. Nowadays research on fractional delay differential equations has become on its initial stage, but the theory of delay differential equations is well developed. For details see references $([1,5,10-13,16,25])$.

The controllability plays a major role in the development of modern mathematical control theory and engineering which has a close connection with structural decomposition, quadratic optimal and so on, see $([14,17,22-24])$. Controllability is a qualitative property in the theory of dynamical system $([2,4,7])$. This means by using some admissible control and some finite time it is possible to steer any initial state of the system to any final state. For finite and infinite dimensional spaces controllability plays a major role, that is, by ordinary and partial differential equations system is represented. Controllability of linear system with delay, nonlinear system with delay and integro-differential systems with delay system has been studied. The work on the controllability of fractional control

\footnotetext{
*Corresponding Author.

†Corresponding Author.

Email addresses: nawazmusarrat@yahoo.com (M. Nawaz), jiangwei@ahu.edu.cn (J. Wei), shengjlny@163.com (J. Sheng), azmatmath@yahoo.com (A.U.K. Niazi), yanglc45@163.com (L. Yang)

Received: 28.05.2018; Accepted: 15.11.2018
} 
system with control delay is discussed by [15] and controllability of nonlinear fractional delay dynamical system has been reported by [20] and the work on the controllability of nonlinear higher order fractional dynamical system is reported by [3] this motivate us to work on the controllability of nonlinear fractional system with control delay. The result for the controllability of fractional linear system is given and then sufficient conditions for controllability of nonlinear fractional systems are established by using the Schauder fixed point theorem. It is also noticed that most of the real systems, such as economic, spaceflight, physiological, biological systems having the phenomena of time delay in the control system.

The main purpose of this paper is to establish the controllability criteria for a fractional nonlinear system with control delay by using fixed point methods, namely the Schauder fixed point theorem. The organization of this paper follows; Section 2 includes some basic definitions, preliminary results and lemmas to prove the controllability of fractional linear system with control delay. In Section 3 we obtain the sufficient condition for controllability of fractional nonlinear system with control delay. In Section 4, an example is given to explain the applicability of the results.

In this paper we study the fractional nonlinear systems with control delay

$$
\left\{\begin{array}{lr}
{ }^{c} D^{\alpha} x(t)=A x(t)+B u(t)+C u(t-\tau)+f(t, x(t), u(t), u(t-\tau)), & t \geq 0, \\
x(0)=x_{0}, & -\tau \leq t \leq 0, \\
u(t)=\psi(t), &
\end{array}\right.
$$

where ${ }^{c} D^{\alpha} x(t)$ denotes $\alpha$ order Caputo fractional derivative of $x(t), 0<\alpha \leq 1, x(t) \in \mathbb{R}^{n}$ is state vector, $u(t) \in \mathbb{R}^{m}$ is control vector, $A \in \mathbb{R}^{n \times n}, \mathrm{~B}, \mathrm{C} \in \mathbb{R}^{n \times m}$ are any matrices, $\tau>0$ is time control delay, and $\psi(t)$ is the initial control function.

\section{Preliminaries and essential lemmas}

This part includes some basic definitions and results used throughout this paper and some lemmas for main results. Let us recall following known definitions. For more detail, see $([18,19])$.

Definition 2.1. Caputo's fractional derivative of order $\alpha(0 \leq m \leq \alpha<m+1)$ for a function $f: R^{+} \rightarrow R$ is defined as

$$
{ }^{c} D^{\alpha} f(t)=\frac{1}{\Gamma(m-\alpha+1)} \int_{0}^{t} \frac{f^{(m+1)}(\theta)}{(t-\theta)^{\alpha-m}} d \theta .
$$

Here $\Gamma(\cdot)$ is the Gamma-Function. The Laplace transform of the Caputo's fractional derivative is

$$
\begin{gathered}
L\{f(t) ;(s)\}=F(s) \\
L\left\{{ }^{c} D^{\alpha} f(t)\right\}(s)=s^{\alpha} F(s)-\sum_{k=0}^{n-1} f(k)\left(0^{+}\right) s^{\alpha-1-k} .
\end{gathered}
$$

Where $f$ be a real or complex-valued function of the variable $t>0$ and $s$ be a real or complex parameter.

Definition 2.2. Riemann-Liouville's fractional integral of order $\alpha>0$ for a function $f: R^{+} \rightarrow R$ is defined as

$$
D^{-\alpha} f(t)=\frac{1}{\Gamma(\alpha)} \int_{0}^{t}(t-\theta)^{\alpha-1} f(\theta) d \theta .
$$

Here $\Gamma(\cdot)$ is the Gamma-Function. 
Remark 2.3. Consider the Mittag-Leffler function

$$
E_{\alpha, \beta}(z)=\sum_{k=0}^{\infty} \frac{z^{k}}{\Gamma(\alpha k+\beta)}, \quad \alpha>0, \beta>0 .
$$

For $\beta=1$, ML function becomes

$$
E_{\alpha}\left(\lambda z^{\alpha}\right)=E_{\alpha, 1}\left(\lambda z^{\alpha}\right)=\sum_{k=0}^{\infty} \frac{\lambda^{k} z^{k \alpha}}{\Gamma(\alpha k+1)}, \quad \lambda, z \in C
$$

For $\beta=1$, the ML function has following property

$$
{ }^{c} D_{+0}^{\alpha} E_{\alpha}\left(\lambda t^{\alpha}\right)=\lambda E_{\alpha}\left(\lambda t^{\alpha}\right)
$$

The Laplace transforms of Mittag-Leffler function is

Particularly, for $\beta=1$

$$
L\left\{t^{\beta-1} E_{\alpha, \beta}\left( \pm a t^{\alpha}\right)\right\}(s)=\frac{s^{\alpha-\beta}}{\left(s^{\alpha} \mp a\right)} .
$$

$$
L\left\{E_{\alpha}\left( \pm a t^{\alpha}\right)\right\}(s)=\frac{s^{\alpha-1}}{\left(s^{\alpha} \mp a\right)} .
$$

Definition 2.4. The system (2.1) is said to be controllable if one can reach any state from any admissible initial state and initial control.

Consider the linear fractional system with control delay of the form

$$
\left\{\begin{array}{lc}
{ }^{c} D^{\alpha} x(t)=A x(t)+B u(t)+C u(t-\tau), & t \geq 0, \\
x(0)=x_{0}, & -\tau \leq t \leq 0, \\
u(t)=\psi(t), &
\end{array}\right.
$$

where ${ }^{c} D^{\alpha} x(t)$ denotes $\alpha$ order Caputo fractional derivative of $x(t), 0<\alpha \leq 1, x(t) \in$ $\mathbb{R}^{n}$ is state vector, $u(t) \in \mathbb{R}^{m}$ is control vector, $A \in \mathbb{R}^{n \times n}, \mathrm{~B}, \mathrm{C} \in \mathbb{R}^{n \times m}$ are any matrices, $\tau>0$ is time control delay, and $\psi(t)$ the initial control function.

Lemma 2.5. The general solution of the system (2.1) is written as

$$
\begin{aligned}
x(t)= & E_{\alpha}\left(A t^{\alpha}\right) x_{0}+\int_{0}^{t}(t-s)^{\alpha-1} E_{\alpha, \alpha} A(t-s)^{\alpha} B u(s) d s \\
& +\int_{-\tau}^{t-\tau}(t-\tau-s)^{\alpha-1} E_{\alpha, \alpha} A(t-\tau-s)^{\alpha} C u(t-\tau) d s .
\end{aligned}
$$

Proof. Consider the linear fractional differential equation with control delay of the form

$$
{ }^{c} D^{\alpha} x(t)=A x(t)+B u(t)+C u(t-\tau),
$$

By taking Laplace transformation of the both sides of equation (2.2) we get

$$
S^{\alpha} X(s)-S^{\alpha-1} x_{0}=A X(s)+L[B u(t)+C u(t-\tau)]
$$

and by the simple calculations we have

$$
X(s)=S^{\alpha-1}\left(S^{\alpha} I-A\right)^{-1} x_{0}+\left(S^{\alpha} I-A\right)^{-1} L[B u(t)+C u(t-\tau)]
$$

by applying inverse Laplace transformation

$x(t)=L^{-1}\{X(s)\}=L^{-1}\left\{S^{\alpha-1}\left(S^{\alpha} I-A\right)^{-1}\right\} x_{0}+L^{-1}\left\{(t)^{\alpha-1} E_{\alpha, \alpha} A(t)^{\alpha} \cdot L(B u(t)+C u(t-\tau))\right\}$

here

$$
=E_{\alpha}\left(A t^{\alpha}\right) x_{0}+t^{\alpha-1} E_{\alpha, \alpha} A(t)^{\alpha} *[(B u(t)+C u(t-\tau)]
$$

now by applying convolution of Laplace transform we get

$t^{\alpha-1} E_{\alpha, \alpha} A(t)^{\alpha} *\left[(B u(t)+C u(t-\tau)]=\int_{0}^{t}(t-s)^{\alpha-1} E_{\alpha, \alpha} A(t-s)^{\alpha}(B u(s) d s+C u(t-\tau)) d s\right.$. 


$$
\begin{gathered}
x(t)=E_{\alpha}\left(A t^{\alpha}\right) x_{0}+\int_{0}^{t}(t-s)^{\alpha-1} E_{\alpha, \alpha} A(t-s)^{\alpha}(B u(s) d s+C u(t-\tau)) d s . \\
x(t)=E_{\alpha}\left(A t^{\alpha}\right) x_{0}+\int_{0}^{t}(t-s)^{\alpha-1} E_{\alpha, \alpha} A(t-s)^{\alpha} B u(s) d s \\
\quad+\int_{-\tau}^{t-\tau}(t-\tau-s)^{\alpha-1} E_{\alpha, \alpha} A(t-\tau-s)^{\alpha} C u(t-\tau) d s .
\end{gathered}
$$

Where $E_{\alpha}\left(A t^{\alpha}\right)$ is the matrix extension of the mentioned Mittag-Leffler function with the following representation:

$$
E_{\alpha}\left(\lambda z^{\alpha}\right)=\sum_{k=0}^{\infty} \frac{\lambda^{k} z^{k \alpha}}{\Gamma(\alpha k+1)} .
$$

by using a property in [16] which can also be written as

$$
\begin{aligned}
x(t)= & E_{\alpha}\left(A t^{\alpha}\right) x_{0}+\int_{0}^{t-\tau}(t-s)^{\alpha-1} E_{\alpha, \alpha} A(t-s)^{\alpha} B u(s) \\
& +(t-\tau-s)^{\alpha-1} E_{\alpha, \alpha} A(t-\tau-s)^{\alpha} C u(t-\tau) d s \\
& +\int_{t-\tau}^{t}(t-s)^{\alpha-1} E_{\alpha, \alpha} A(t-s)^{\alpha} B u(s) d s \\
& +\int_{-\tau}^{0}(t-\tau-s)^{\alpha-1} E_{\alpha, \alpha} A(t-\tau-s)^{\alpha} C \psi(s) d s .
\end{aligned}
$$

And consider the corresponding nonlinear system represented in system (1.1) its solution can be written as

$$
\begin{aligned}
x(t)= & E_{\alpha}\left(A t^{\alpha}\right) x_{0}+\int_{0}^{t}(t-s)^{\alpha-1} E_{\alpha, \alpha} A(t-s)^{\alpha} B u(s)+C \psi(s) d s \\
& +\int_{0}^{t}(t-s)^{\alpha-1} E_{\alpha, \alpha} A(t-s)^{\alpha} f(s, x(s), u(s), u(s-\tau)) d s .
\end{aligned}
$$

Definition 2.6. We call the set as in [15] $R\left(x_{0}, \psi\right)=\left\{\nu \mid\right.$ there exists $t_{1}>0, u(t) \in C^{l-1}$, such that the solution of the system (1.1) $x\left(t, x_{0}, \psi\right)$ satisfies that $\left.x\left(t_{1}, x_{0}, \psi\right)=\nu\right\}$ the reachable set of (2.1) $x(0)=x_{0}$ and $u(t)=\psi(t),-\tau \leq t \leq 0$.

Lemma 2.7 ([15]). The linear fractional control system with control delay for system (2.1) is controllable if and only if

$$
\operatorname{rank}\left[B, A B, A^{2} B, \ldots A^{n-1} B, C, A C, A^{2} C, A^{3} C, \ldots, A^{n-1} C\right]=n .
$$

\section{Main results}

Consider a nonlinear control system with control delay represented by a form of fractional differential equation in system (1)

Where ${ }^{c} D^{\alpha} x(t)$ denotes $\alpha$ order-Caputo fractional derivative of $x(t), 0<\alpha \leq 1 . x(t) \in$ $\mathbb{R}^{n}$ is state vector, $u(t) \in \mathbb{R}^{m}$ is control vector, $A \in \mathbb{R}^{n \times n}, \mathrm{~B}, \mathrm{C} \in \mathbb{R}^{n \times m}$ are any matrices, and $A$ is a $n \times n$ matrix and $B$ is a $n \times m$ matrix and $C$ is also a $n \times m$ matrix and $f: J \times \mathbb{R}^{n} \times \mathbb{R}^{m} \times \mathbb{R}^{m} \rightarrow \mathbb{R}^{n}$ is continuous $\tau>0$ is time control delay, and $\psi(t)$ the initial control function. Let us introduce the following notation, denote $Q$ as Banach space of continuous $\mathbb{R}^{n} \times \mathbb{R}^{m} \times \mathbb{R}^{m}$ valued functions defined on the interval $J$ with the norm

$$
\|(x, u)\|=\|x\|+\|u\|
$$

where

$$
\|x\|=\sup \{|x(t)|: t \in J\},
$$


and

$$
\|u\|=\sup \{|u(t)|: t \in J\},
$$

that is $Q=C_{n(J)} \times C_{m(J)} \times C_{m(J)}$ where $C_{n(J)}$ is the Banach space of continuous $\mathbb{R}^{n}$ valued function defined on the interval $J$ with the sup norm for each $(z, \nu) \in Q$ the solution of the above system (1.1) is

$$
\begin{aligned}
x(t)= & E_{\alpha}\left(A t^{\alpha}\right) x_{0}+\int_{0}^{t}(t-s)^{\alpha-1} E_{\alpha, \alpha} A(t-s)^{\alpha} B u(s) \\
& +C \psi(s) d s+\int_{0}^{t}(t-s)^{\alpha-1} E_{\alpha, \alpha} A(t-s)^{\alpha} f(t, x(t), u(t), u(t-\tau)) d s .
\end{aligned}
$$

Now we obtain our main result on the controllability of the nonlinear fractional control delay system (1.1), for this we take

$$
p=\left(x, u, u^{\prime}\right) \in \mathbb{R}^{n} \times \mathbb{R}^{m} \times \mathbb{R}^{m}
$$

and let

$$
|p|=|x|+|u|+\left|u^{\prime}\right|
$$

for $t \in[0, \tau]=J$ where $u(t)=\psi(t)$ at $-\tau \leq t \leq 0$.

Theorem 3.1. Let the continuous function $f$ satisfy the condition

$$
\lim _{|p| \rightarrow \infty} \frac{|f(t, p)|}{|p|}=0
$$

uniformly in $t \in J$ and suppose that the nonlinear system (1.1) is controllable on $J$.

Proof. Let $x \in \mathbb{R}^{m}$ and $Q$ be the Banach space of all functions where $x$ is continuous and $u$ is an admissible control function, with norm defined by

$$
\|(x, u)\|=\|x\|+\|u\|,
$$

Define the operator

$$
T: Q \rightarrow Q
$$

by

$$
T(x, u)=(z, \nu)
$$

for $t \in[0, \tau]$

$$
W=\int_{0}^{t}(t-s)^{\alpha-1}\left[E_{\alpha, \alpha} A(t-s)^{\alpha} B\right]\left[E_{\alpha, \alpha} A(t-s)^{\alpha} B\right]^{*} d s
$$

where

$$
\begin{aligned}
& \nu(t)=B^{*} E_{\alpha, \alpha} A^{*}(t-s)^{\alpha} w^{-1}\left[x_{1}-E_{\alpha}\left(A t^{\alpha}\right) x_{0}\right] \\
& -\int_{0}^{t}(t-s)^{\alpha-1} E_{\alpha, \alpha} A(t-s)^{\alpha} f(s, x(s), u(s), u(s-\tau)) d s, \\
& z(t)=E_{\alpha}\left(A t^{\alpha}\right) x_{0}+\int_{0}^{t}(t-s)^{\alpha-1} E_{\alpha, \alpha} A(t-s)^{\alpha} B u(s)+C \psi(s) d s \\
& +\int_{0}^{t}(t-s)^{\alpha-1} E_{\alpha, \alpha} A(t-s)^{\alpha} f(s, x(s), u(s), u(s-\tau)) d s, \\
& a_{1}=\sup \left\|E_{\alpha, \alpha} A(t-s)^{\alpha}\right\|, \quad a_{2}=\sup \left\|E_{\alpha}\left(A t^{\alpha}\right) x_{0}\right\|, \\
& a=\max \left\{a_{1} b^{\alpha} \alpha^{-1}\|B\|, 1\right\}, \quad c_{1}=6 a_{1}^{2} b^{\alpha}\left\|B^{*}\right\|\left\|w^{-1}\right\| \alpha^{-1}, \\
& c_{2}=6 a_{1} b^{\alpha} \alpha^{-1}, \quad c=\max \left\{c_{1}, c_{2}\right\}, \\
& d_{1}=6 a_{1}\left\|B^{*}\right\|\left\|w^{-1}\right\|\left[\left|x_{1}\right|+a_{2}\right], \quad d_{2}=6 a_{2}, \quad d=\max \left\{d_{1}, d_{2}\right\} \\
& \sup |f|=\sup |f(s, x(s), u(s), u(s-\tau))| \text { where } s \in J
\end{aligned}
$$


Then

$$
\begin{aligned}
|\nu(t)| & \leq\left\|B^{*}\right\| a_{1}\left\|w^{-1}\right\|\left[\left|x_{1}\right|+a_{2}+b^{\alpha} a_{1} \alpha^{-1} \sup |f|\right] \\
& \leq\left[\frac{d_{1}}{6 a}+\frac{c_{1}}{6 a} \sup |f|\right] \\
& \leq \frac{1}{6}[d+c \sup |f|]
\end{aligned}
$$

for $t \in J$,and

$$
\begin{aligned}
|z(t)| & \leq a_{2}+\frac{a_{1} b^{\alpha}\|B\|}{6 a \alpha}[d+c \sup |f|]+\frac{a_{1} b^{\alpha}}{\alpha} \sup |f| \\
& \leq \frac{d}{6}+\frac{1}{6}[d+c \sup |f|]+\frac{c}{6} \sup |f| \\
& \leq \frac{d}{6}+\frac{c}{6} \sup |f| \text { for } t \in J .
\end{aligned}
$$

The function $f$ satisfies the following conditions by proposition in [6], $f$ satisfied the following conditions: for each pair of positive constants $c$ and $d$, there exists a positive constant $r$, such that if $|p| \leq r$, then

$$
c|f(t, p)|+d \leq r \text { for all } t \in J .
$$

For given $c$ and $d$, if $r$ is a constant then the inequality in (3.1) is satisfied. Then any $r_{1}$ such that $r<r_{1}$ will also satisfy the inequality. Now, take $c$ and $d$ as given above and let $r$ be chosen so that the implication in (3.1) inequality is satisfied. Therefore if

$$
\|x\| \leq r / 3
$$

and

then

$$
\|u\| \leq r / 3
$$

It follows that

$$
|x(s)|+|u(s)|+|u(s-\tau)| \leq r \text { for all } s \in J .
$$

$$
d+c \sup |f| \leq r \text { for } s \in J
$$

And therefore, $|\nu(t)| \leq r / 6 a$ for all $t \in J$, and hence $\|\nu\| \leq r / 6 a$. It follows that $|z(t)| \leq$ $r / 3+r / 3$ for all $t \in J$, and hence that $\|z\| \leq r / 3$ thus we have proved that if

$$
Q(r)=\{(x, u) \in Q /\|x\| \leq r / 3, \quad\|u\| \leq r / 3\}
$$

then p maps $Q(r)$ itself. Our objective is to prove that $T$ has a fixed point since $f$ is continuous, it follows $T$ is continuous. Let $Q^{\prime}$ be a bounded subset of $Q$. Consider a sequence $\left\{z_{i}, \nu_{i}\right\}$ contained in $T\left(Q^{\prime}\right)$ where we let

$$
\left\{z_{i}, \nu_{i}\right\}=T\left\{x_{i}, u_{i}\right\}
$$

for some $\left(x_{i}, u_{i}\right) \in Q^{\prime}$ for $i=1,2,3 \ldots$ Since $f$ is continuous, then

$$
|f(s, x(s), u(s), u(s-\tau))|
$$

is uniformly bounded for all $s \in J$ and all $i=1,2,3 \ldots$, it follows that $\left\{z_{i}, \nu_{i}\right\}$ is a bounded sequence in $Q$. Hence $\left\{\nu_{i}(t)\right\}$ is equicontinuous and a uniformly bounded sequence on $\left[-\tau, t_{1}\right]$. Since each $\nu_{i}(t)$ has both right and left limits at $t=0$ and $t=t_{1}-\tau$, we can apply Ascoli's theorem on $\left[0, t_{1}-\tau\right]$ to the sequence $\left\{\nu_{i}(t)\right\}$. Therefore there exist a subsequence of $\left\{\nu_{i}(t)\right\}$ which converges uniformly to a continuous function on $\left[0, t_{1}-\tau\right]$. Now apply Ascoli's theorem again to this subsequence to obtain a further subsequence which converges uniformly to a continuous function on $\left[t_{1}-\tau, t_{1}\right]$ a further application of Ascoli's theorem yields a further subsequence of $\left\{z_{i}, \nu_{i}\right\}$ which converges in $Q$ to some $\left(x_{0}, u_{0}\right)$. It follows that $T\left(Q^{\prime}\right)$ is sequentially compact, hence the closure is sequentially compact. Thus $T$ is completely continuous. Since $Q(r)$ is closed, bounded and convex, 
the Schauder fixed-point theorem implies that $T$ has a fixed point $(x, u) \in Q(r)$. It follows that

$$
\begin{aligned}
x(t)= & E_{\alpha}\left(A t^{\alpha}\right) x_{0}+\int_{0}^{t}(t-s)^{\alpha-1} E_{\alpha, \alpha} A(t-s)^{\alpha} B u(s)+C u(t-\tau) d s \\
& +\int_{0}^{t}(t-s)^{\alpha-1} E_{\alpha, \alpha} A(t-s)^{\alpha} f(s, x(s), u(s), u(s-\tau)) d s .
\end{aligned}
$$

For $t \in J$, and $u(t)=\psi(t)$ for $t \in[0, t]$. Hence $x(t)$ is a solution of system (1.1) and

$$
\begin{aligned}
x(t)= & E_{\alpha}\left(A t^{\alpha}\right) x_{0}+\int_{0}^{t}(t-s)^{\alpha-1} E_{\alpha, \alpha} A(t-s)^{\alpha} B B^{*} E_{\alpha, \alpha} A^{*}(t-s)^{\alpha} w^{-1}\left[x_{1}-E_{\alpha}\left(A t^{\alpha}\right) x_{0}\right. \\
& -\int_{0}^{t}(t-s)^{\alpha-1} E_{\alpha, \alpha} A(t-s)^{\alpha} f(s, x(s), u(s), u(s-\tau)) d s \\
& +\int_{0}^{t}(t-s)^{\alpha-1} E_{\alpha, \alpha} A(t-s)^{\alpha} f(s, x(s), u(s), u(s-\tau)) d s=x_{1} .
\end{aligned}
$$

so the system (1.1) is controllable on $J$.

Corollary 3.2. Let the continuous function $f$ be bounded on $J \times \mathbb{R}^{n} \times \mathbb{R}^{m} \times \mathbb{R}^{m}$, suppose system (2.1) is controllable on $J$ then system (1.1) is controllable on $J$.

\section{Examples}

In this section, we will apply the results we obtained in the previous section for nonlinear fractional system with control delay.

\section{Example 4.1.}

$$
{ }^{c} D^{\alpha} x(t)=A x(t)+B u(t)+C u(t-\tau)+f(t, x(t), u(t), u(t-\tau)),
$$

$x(0)=x_{0}$ for $t \in J$ and $0<\alpha<1$ where $A=\left(\begin{array}{cc}1 & 2 \\ -2 & 0\end{array}\right), B=\left(\begin{array}{ll}1 & 0 \\ 0 & 2\end{array}\right), C=\left(\begin{array}{ll}1 & 0 \\ 0 & 1\end{array}\right)$ and $f(t, p)=\left(\begin{array}{c}\frac{x_{1}}{1+x_{2}^{2}+u^{2}(t)+u^{2}(t-\tau)} \\ \frac{x_{2}}{1+x_{1}^{2}+u^{2}(t)+u^{2}(t-\tau)}\end{array}\right)$. Here $x(t)=\left(\begin{array}{c}x_{1}(t) \\ x_{2}(t)\end{array}\right)$

$$
W=\int_{0}^{t}(t-s)^{\alpha-1}\left[E_{\alpha, \alpha} A(t-s)^{\alpha} B\right]\left[E_{\alpha, \alpha} A(t-s)^{\alpha} B\right]^{*} d s
$$

$\alpha=\frac{1}{2}$

$$
\begin{gathered}
{\left[E_{\alpha, \alpha} A(t-s)^{\alpha}\right]=\frac{1}{\sqrt{\pi}}\left(\begin{array}{cc}
1 & 0 \\
0 & 1
\end{array}\right)-\left(\begin{array}{cc}
1 & 2 \\
-2 & 0
\end{array}\right)(1-s)^{\frac{1}{2}}} \\
{\left[E_{\alpha, \alpha} A^{*}(t-s)^{\alpha}\right]=\frac{1}{\sqrt{\pi}}\left(\begin{array}{ll}
1 & 0 \\
0 & 1
\end{array}\right)-\left(\begin{array}{cc}
1 & -2 \\
2 & 0
\end{array}\right)(1-s)^{\frac{1}{2}}} \\
B B^{*}=\left(\begin{array}{ll}
1 & 0 \\
0 & 4
\end{array}\right) \\
w[0,1]=\int_{0}^{1}(1-s)^{\frac{-1}{2}}\left[\frac{1}{\sqrt{\pi}}\left(\begin{array}{ll}
1 & 0 \\
0 & 1
\end{array}\right)-\left(\begin{array}{cc}
1 & 2 \\
-2 & 0
\end{array}\right)(1-s)^{\frac{1}{2}}\right]\left(\begin{array}{ll}
1 & 0 \\
0 & 4
\end{array}\right) \\
\times\left[\frac{1}{\sqrt{\pi}}\left(\begin{array}{cc}
1 & 0 \\
0 & 1
\end{array}\right)-\left(\begin{array}{cc}
1 & -2 \\
2 & 0
\end{array}\right)(1-s)^{\frac{1}{2}}\right] d s \\
\approx\left(\begin{array}{cc}
\frac{-2-2 \sqrt{\pi}}{\pi}-\frac{34}{3} & \frac{4}{3}-\frac{6}{\sqrt{\pi}} \\
-\frac{6}{\sqrt{\pi}}+\frac{4}{3} & -\frac{8}{\sqrt{\pi}}-\frac{8}{3}
\end{array}\right)
\end{gathered}
$$

As $w[0,1]$ is non singular so the nonlinear function $f(t, p)$ is bounded and continuous and all conditions stated in theorem 3.1 are satisfied. Hence the fractional system is controllable on $[0,1]$. 
Remark 4.2. The impulsive effect condition will be difficult, we can consider it in future work.

Acknowledgment. We would like to thank the anonymous referees for their comments and suggestions which have considerably improved the quality and presentation of this paper. This research had been supported by National Natural Science Foundation of China (no.11371027, no.11471015 and no.11601003).

\section{References}

[1] J.O. Alzabut, Existence of periodic solutions of a type of nonlinear impulsive delay differential equations with a small parameter, J. Nonlinear Math. Phys. 15, 13-21, 2008.

[2] K. Balachandran, J.P. Dauer, Controllability of nonlinear systems via fixed-point theorems, J. Optim. Theory Appl. 53 (3), 345-352, 1987.

[3] K. Balachandran, V. Govindaraj, L. Rodriguez-Germa, and J.J. Trujillo, Controllability of nonlinear higher order fractional dynamical systems, Nonlinear Dynam. 156, 33-44, 2013.

[4] K. Balachandran, J.Y. Park, and J.J. Trujillo, Controllability of nonlinear fractional dynamical systems, Nonlinear Anal. 75 (4), 1919-1926, 2012.

[5] L. Dai, Singular Control Systems, Springer, 1989.

[6] J.P. Dauer, Nonlinear Perturbations of Quasi-Linear Control Systems, J. Math. Anal. Appl. 54, 717-725, 1976.

[7] J.P. Dauer, R.D. Gahl, Controllability of nonlinear delay systems, J. Optimiz. Theory Appl. 21 (1), 59-70, 1977.

[8] Park, K. Diethel, The analysis of fractional differential equations, Lect. Notes Math., 2010.

[9] J. Hale, Introduction to functional differential equations, Springer Verlag, 1992.

[10] W. Jiang, The degeneration differential systems with delay, Anhui University Press, 1998.

[11] W. Jiang, Eigenvalue and stability of singular differential delay systems, J. Math. Anal. Appl. 297, 305-316, 2004.

[12] W. Jiang, Function-controllability of nonlinear singular delay differential control systems, Acta Math. Sinica (Chin. Ser.) 49 (5), 1153-1162, 2006.

[13] W. Jiang, On the solvability of singular differential delay systems with variable coefficients, Int. J. Dyn. Syst. Differ. Equ. 4, 245-249, 2008.

[14] W. Jiang, The constant variation formulae for singular fractional differential systems with delay, Comput. Math. Appl. 59 (3), 1184-1190, 2010.

[15] W. Jiang, The controllability of fractional control systems with control delay, Comput. Math. Appl. 64, 3153-3159, 2012.

[16] W. Jiang, On the interval controllability of fractional systems with control delay, J. Math. Res. 9 (5), 87, 2017.

[17] W. Jiang and W. Song, Controllability of singular systems with control delay, Automatica J. IFAC 37, 1873-1877, 2001.

[18] A.A. Kilbas, H.M. Srivastava, and J.J. Trujillo, Theory and applications of fractional differential equations, Elsevier Science Publishers, 204, 2006.

[19] K.S. Miller and B. Ross, An introduction to the fractional calculus and differential equations, John Wiley and Sons, 1993.

[20] R.J. Nirmala, K. Balachandran, L.R. Germa, and J.J. Trujillo, Controllability of nonlinear fractional delay dynamical systems, Rep. Math. Phys. 77 (1), 87-104, 2016.

[21] I. Podlubny, Fractional differential equations, Academic Press, San Diego, 1999. 
[22] S.H. Saker and J.O. Alzabut, Periodic solutions global attractivity and oscillation of an impulsive delay host macroparasite model, Math. Comput. Model. 45, 531-543, 2007.

[23] G.T. Stamov and J.O. Alzabut, Almost periodic solutions in the PC-space for uncertain impulsive dynamical systems, Nonlinear Anal. 74, 4653-4659, 2011.

[24] G.T. Stamov, I.T. Stamov and J.O. Alzabut, Existence of almost periodic solutions for strongly stable nonlinear impulsive differential difference equations, Nonlinear Anal. Hybrid Syst. 6, 818-823, 2012.

[25] I.T. Stamov, G.T. Stamov, and J.O. Alzabut, Global exponential stability for a class of impulsive BAM neural networks with distributed delays, Appl. Math. Inf. Sci. 7, 1539-1546, 2013.

[26] Z.X. Zheng, Theory of functional differential equations, Anhui Education Press, 1994.

[27] X.F. Zhou, W. Jiang, and L.G. Hu, Controllability of a fractional linear time-invariant neutral dynamical system, Appl. Math. Lett. 26, 418-424, 2013. 